\title{
Modelo para el gobierno y la gestión de las tecnologías de la información para una organización inteligente
}

\section{Model for government and information technology management for a smart organization}

CANEDO-MONTOYA, Enrique Daniel†', CANEDO-MONTOYA, Gerardo Miguel, LÓPEZ-MEJÍA, Nancy Maria y CANEDO-ROMERO, Gerardo Enrique

\author{
Universidad Politécnica de Juventino Rosas, México. \\ Universidad de Guanajuato, México. \\ ID $1^{\mathrm{er}}$ Autor: Enrique Daniel, Canedo-Montoya / ORC ID: 0000-0002-8062-9567, SNI CONACYT ID: 520884 \\ ID $1^{\mathrm{er}}$ Coautor: Gerardo Miguel, Canedo-Montoya / ORC ID: 0000-0002-4036-2394, SNI CONACYT ID: 994258 \\ ID $2^{\text {do }}$ Coautor: Nancy Maria, López-Mejía / ORC ID: 0000-0003-4508-3869, SNI CONACYT ID: 1048798 \\ ID $3^{\text {er }}$ Coautor: Gerardo Enrique, Canedo-Romero / ORC ID: 0000-0001-6624-185X, SNI CONACYT ID: 994269
}

\begin{abstract}
Resumen
En el foro económico mundial en Davos en el 2016 se formalizo el concepto de industria 4.0 lo que marcaba una trasformación de la industria digital a la industria inteligente. Uno de los problemas más apremiantes es el modelado de la industria inteligente como una organización ya que los estándares son lineales y se requieren modelos circulares para la detección continua de las tendencias en su área de impacto. En este trabajo se propone un modelo para las organizaciones inteligentes que permita resolver el anterior problema.
\end{abstract}

Organización inteligente, Modelo innovador, Circulo de Valor

\begin{abstract}
The world economic forum in Davos in 2016 formalised the concept of Industry 4.0, marking a transformation from the digital industry to the smart industry. One of the most pressing problems is the modeling of the intelligent industry as an organization since the standards are linear and circular models are required for the continuous detection of trends in their impact area. This work proposes a model for smart organizations to solve the previous problem.
\end{abstract}

Smart organization, Innovative model, Circle of value

Citación: CANEDO-MONTOYA, Enrique Daniel, CANEDO-MONTOYA, Gerardo Miguel, LÓPEZ-MEJÍA, Nancy Maria y CANEDO-ROMERO, Gerardo Enrique. Modelo para el gobierno y la gestión de las tecnologías de la información para una organización inteligente. Revista de Tecnologías de la Información. 2020. 7-22:1-4.

\footnotetext{
$\uparrow$ Investigador contribuyendo como primer autor.
} 


\section{Introducción}

En el foro económico mundial en Davos del año 2016 [1] estuvo dedicado a introducir el concepto de industria 4.0. La premisa fundamental de la industria 4.0 es la detección continua de las tendencias en su nicho de mercado que permita su ajuste en el menor tiempo posible a esta situación. La herramienta que permite lo anterior es la inteligencia artificial. En el presente trabajo se considera un concepto más general como lo es la organización inteligente. En este trabajo se propone un modelo innovador que permita generar conocimiento para ajustar las estrategias de la organización a las tendencias sobre las preferencias de los usuarios y poder ajustar la estrategia para satisfacer esas demandas.

Con este modelo se pretende que apoyado en la innovación a nivel estratégico las organizaciones respondan en corto tiempo a los cambios del mercado que cada vez exigen productos y servicios personalizados.

\section{Concepto de organización inteligente}

Se puede describir el concepto de una organización inteligente de la siguiente manera [2]: La Organización Inteligente es una entidad que detecta la información de su entorno interno y de las tendencias de su entorno externo para la generación del conocimiento que permita la toma de decisiones a nivel estratégico para el cumplimiento de los objetivos de la organización.

Uno de los pilares de la organización inteligente es la innovación, desde la innovación organizacional pasando por la organización de sistemas de información y la innovación tecnológica.

La generación del conocimiento implica a la innovación inteligente en dos aspectos:

- Interacción. El paradigma innovador de las relaciones humanas con la automatización de los procesos.

- Interoperabilidad. La aplicación de la inteligencia artificial distribuida para la automatización de los procesos (ver figura $1)$.

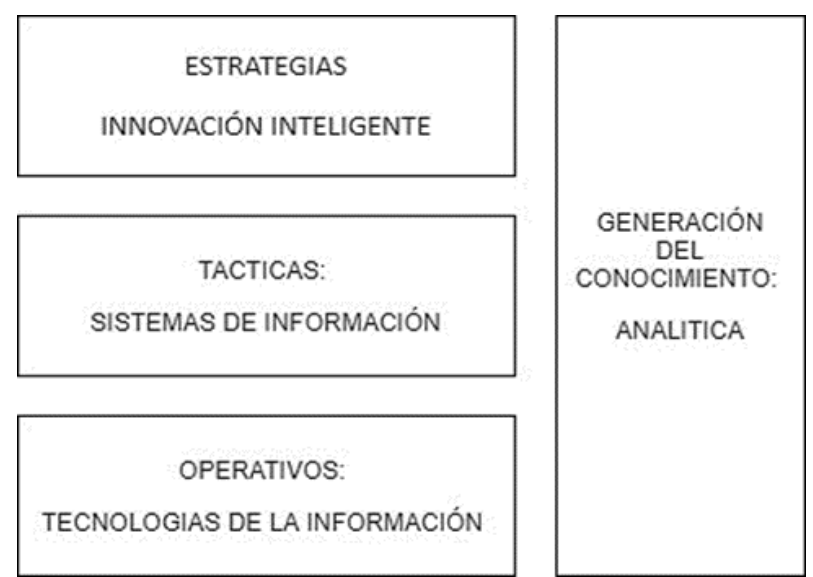

Figura 1 Arquitectura de una Organización Fuente: elaboración propia [Draw.io]

\section{Generación del conocimiento: analitica}

Se han realizado esfuerzos para modelar las organizaciones inteligentes $[3,4]$.

El modelo propuesto en este trabajo está enfocado al concepto de interoperabilidad de los procesos dentro de la organización inteligente.

\section{Definición del problema}

Los modelos de organización existentes como es la cadena de valor [5] [ver figura 2] y el modelo canvas [6] [ver figura 3] son lineales donde el usuario se encuentra al final del modelo, esta situación dificulta el modelado de la organización inteligente ya que el usuario participa en la organización desde el inicio, ya que es el que establece las tendencias de mercado.

\begin{tabular}{|c|c|c|}
\hline PROCESO 1 & $\cdots$ & PROCESO N \\
\hline \multicolumn{2}{|c|}{ RECURSOS HUMANOS } \\
\hline \multirow{2}{*}{ RECURSOS MATERIALES } \\
\hline
\end{tabular}

Figura 2 Modelo de cadena de valor

Fuente: elaboración propia [Draw.io]

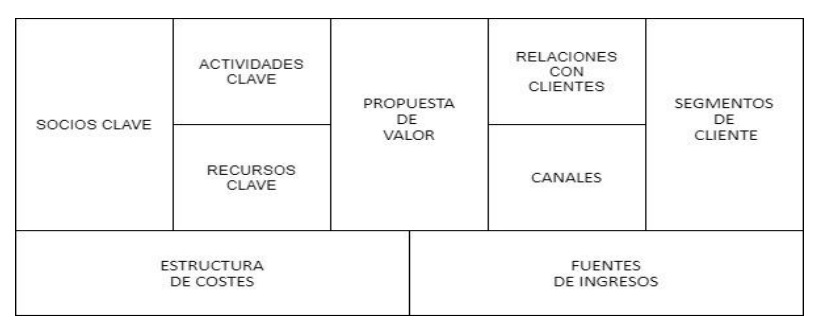

Figura 3 Modelo Canvas

Fuente: Adaptado de [6] [Draw.io]

\section{Fuentes de ingresos}

CANEDO-MONTOYA, Enrique Daniel, CANEDO-MONTOYA, Gerardo Miguel, LÓPEZ-MEJÍA, Nancy Maria y CANEDO-ROMERO, Gerardo Enrique. Modelo para el gobierno y la gestión de las tecnologías de la información para una organización inteligente. Revista de Tecnologías de la Información. 2020 
Por otro lado, los estándares como COBIT 5 [7] y IT4IT [8] también son de naturaleza lineal por lo tanto se dificulta estandarizar las tecnologías de la información. Los modelos de organización y los estándares no consideran a la innovación [3] como un pilar de la estrategia.

\section{Modelo propuesto}

El presente modelo trata de resolver la problemática descrita en el párrafo anterior $\mathrm{y}$ está basado en las siguientes premisas:

- La innovación como pilar de la estrategia de la organización.

- Adaptación del estándar IT4IT para la detección continúa de la información del entorno y generar conocimiento para la toma de decisiones en nivel estratégico (ver Figura 4).

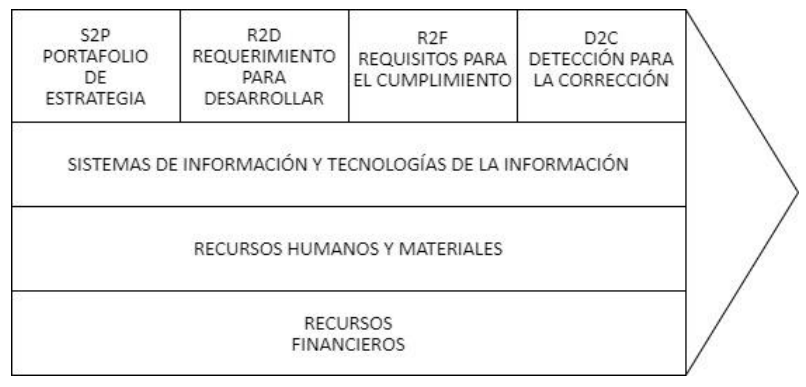

\section{Figura 4 IT4IT}

Fuente: adaptado de [8] [Draw.io]

- La incorporación de la inteligencia artificial distribuida, conocida como sistemas multi agente que permita la automatización de los procesos en la organización.

\section{Descripción del modelo}

El presente modelo se aleja del modelo clásico piramidal dirigiéndose más a un modelo "plano" con este cambio se definen la conectividad horizontal y vertical de los procesos de la organización.

El modelo considera a lo que se llamara la innovación inteligente a nivel organizacional de los procesos de los sistemas de información y de las operaciones a nivel de las tecnologías de la información.

Se adapta el modelo IT4IT para que permita la estandarización de los sistemas y tecnologías de la información de forma cíclica teniendo al usuario como centro de acción. En la siguiente figura se muestra el modelo (ver Figura $5)$.

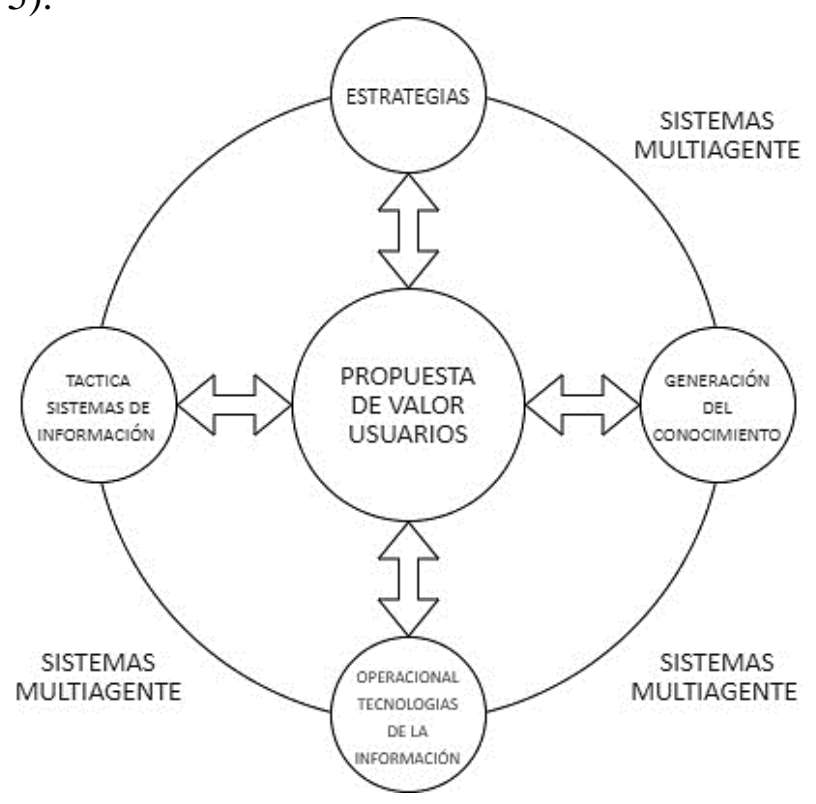

Figura 5 Modelo propuesto de circulo de valor Fuente: Elaboración propia [Draw.io]

\section{Conclusiones}

Este modelo innovador para el gobierno y la gestión de las tecnologías de la información para una organización inteligente permite establecer las políticas de innovación a nivel estratégico y de forma permanente. También este modelo permite la definición de los sistemas y tecnologías de la información desde el nivel estratégico por lo tanto se considera que el objetivo del trabajo se cumplió satisfactoriamente. Como trabajo futuro se pretende introducir la simulación por sistemas multi agente para determinar la verificación y valides para una organización dada.

\section{References}

[1] World Economic Forum 2016 in Davos https://www.weforum.org/agenda/2016/0 1/a-recap-of-davos-2016 Revisado y actualizado 4 de abril del 2020

[2] Choo C.W., La Organización Inteligente: El empleo de la información para dar significado, crear conocimiento y tomar decisiones, Oxford University Press, 1999. 
[3] Azar G., and F. Ciabuschi, (2017), Organizational innovation, technological innovation, and export performance: the effects of innovation radicalness and extensiveness, International Business Review, ELSEVIER.

[4] Caetano A., G. Antunes, J. Pombinho, M. Bakhshandeh, J. Granjo, J. Brobinha, and M. Mira da Silva, (2016), Representation and analisis of Enterprise models with semantic techniques: an application to ArchiMate e3value and business model canvas, Knowl Inf Syst, Springer-Verlag.

[5] Porter, Michael, (1987) "ventaja competitiva. Creación y sostenimiento de un desempeño superior" Mexico: Editorial Compañía Editorial Continental 1982.

[6] Osterwalder A., and Y. Pigneur, (2011), Generación de modelos de negocio, DEUSTO.

[7] ISACA, (2012), Un marco de Negocio para el Gobierno y la Gestión de las TI de la empresa, COBIT 5, ISACA.

[8] TOG (2015), The Open Group IT4IT Reference Architecture, Version 2.0, The Open Group 